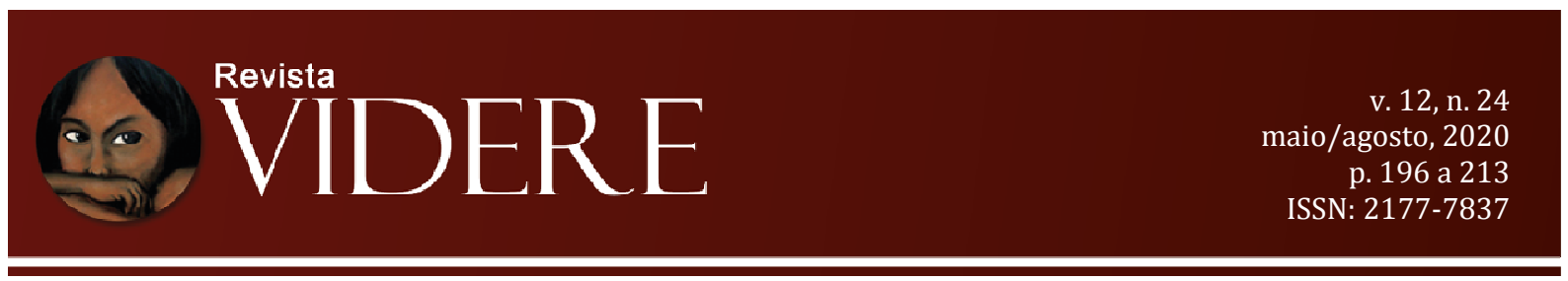

\title{
MEDIACIÓN PENAL JUVENIL EN CHILE: LÍMITES Y POSIBILIDADES $^{87}$
}

\section{JUVENILE CRIMINAL MEDIATION IN CHILE: LIMITS AND POSSIBILITIES}

\author{
Rosane Teresinha Carvalho Porto \\ Doutora em Direito (UNISC), Brasil \\ Mestre em Direito (UNISC), Brasil \\ Professora da Universidade de Santa Cruz do Sul \\ (UNISC), Brasil \\ E-mail: rosaneporto@unisc.br \\ OrcID: https://orcid.org/0000-0002-1875-5079
}

\author{
Rodrigo Cristiano Diehl \\ Doutorando em Direito (UNISC), Brasil \\ Mestre em Política Social e Serviço Social \\ (UFRGS), Brasil \\ E-mail: rodrigocristianodiehl@live.com \\ OrcID: http://orcid.org/0000-0002-4869-3112
}

RESUMEN: El presente estudio tiene como objetivo contextualizar sobre la mediación penal juvenil en Chile, siendo esta una de las prácticas de Justicia Restaurativa. El tema se considera actual debido a los desafíos y perspectivas basadas en el abolicionismo penal de la criminología crítica de Hulsman y Nils Christie. En este contexto la pregunta es: ¿cuáles son los límites y las posibilidades de la implementación de la mediación penal juvenil en Chile en cuanto una de las prácticas de Justicia Restaurativa? En términos concluyentes, en cuanto a la legislación juvenil chilena, es necesario revisarla y reexaminarla, porque la falta de especialización para jueces y fiscales, así como el paradigma punitivo con respecto a los adolescentes, dificultan la conexión y el diseño institucional de implementación y aplicación de las prácticas de Justicia Restaurativa en el país a través de una política pública. En materia metodológica, se utiliza el materialismo dialéctico como método de aproximación y la técnica de investigación documental y bibliográfica.

PALABRAS CLAVE: Abolicionismo penal. Adolescentes. Chile. Justicia Restaurativa.

ABSTRACT: The objective of this study is to contextualize juvenile criminal mediation in Chile, this being one of the practices of Restorative Justice. The topic is considered current

\footnotetext{
${ }^{87}$ Resultado parcial de la pasantía postdoctoral por la Universidad La Salle (RS), Brasil. Asesor: Dr. Daniel Achutti. Doctor en Ciencias Penales por la PUCRS (2012), con un período de prácticas en la Universidad de Leuven - Bélgica (2010/2011). Profesor Permanente de Maestría y Doctorado en Derecho y del Curso de Derecho de la Universidad La Salle (RS). Coordinador del Área de Derecho y Política de la Universidad La Salle (RS). Académico visitante en la Università IUAV di Venezia, Italia (2014-2015), y en el Leuven Institute of Criminology - Universidad de Leuven, Bélgica (2013-2014 y 2017-2018). Abogado. Autor del libro: Justicia Restaurativa y Abolicionismo Penal, $2^{a}$ ed., ed. Saraiva ( $2^{\circ}$ lugar en la categoría de Derecho - Premio Jabuti 2015). Tiene experiencia en el área de Derecho, con énfasis en Derecho Penal y Procesal Penal, Criminología, Justicia Restaurativa y Justicia Criminal.
} 
due to the challenges and perspectives based on the criminal abolitionism of the critical criminology of Hulsman and Nils Christie. In this context, the question is: what are the limits and possibilities of the implementation of juvenile criminal mediation in Chile as one of the practices of Restorative Justice? In conclusive terms, regarding Chilean youth legislation, it is necessary to review and reexamine it, because the lack of specialization for judges and prosecutors, as well as the punitive paradigm regarding adolescents, hinder the connection and the institutional design of implementation and application of Restorative Justice practices in the country through a public policy. In methodological matters, dialectical materialism is used as an approximation method and the documentary and bibliographic research technique.

KEYWORDS: Criminal abolitionism. Teenagers. Chile. Restorative Justice.

\section{Introducción}

Antes de trabajar en la multidimensionalidad de la Justicia Restaurativa basada en principios, prácticas y la comunidad, vale la pena señalar que esta justicia hipotéticamente no pertenece al Estado, que en general encarcela, institucionaliza lo que quiere tener como instrumento de control social. La justicia restaurativa parte de las relaciones humanas, del sentido de pertenencia que da sentido a la construcción y convivencia comunitaria. Por lo tanto, el tema de esta comunidad ve el conflicto como oportunidades, ya que la resolución de sus conflictos tendrá lugar de manera coherente. En otras palabras, con el sentimiento de pertenencia y un sentido de comunidad, los sujetos entienden que el manejo del conflicto social se resuelve en amplias esferas democráticas y no en las viejas estructuras, otorgadas por las instituciones actuales que retroalimentan verticalmente y con el efecto de la cultura legal tradicional y conservador en Chile.

En el contexto internacional, su regulación fue: por los actos del Consejo Económico y Social (CES) de las Organizaciones de las Naciones Unidas (ONU): Resolución CES/UN 1.999/26, de 28 de julio de 1999: que prevé la "Preparación y aplicación de la justicia restaurativa y medidas de mediación en materia de justicia penal"; Resolución CES/ONU 2000/14 de 27 de julio de 2000: "Principios básicos sobre el uso de programas de Justicia Restaurativa en materia penal". ONU No. 2,002/12, 24 de julio de 2002: luego definió "Principios básicos para el uso de programas de Justicia Restaurativa en asuntos penales" (FLORES; ROLIANO, 2016).

Dentro de la justicia juvenil, la JR es especialmente valorada debido a la consistencia entre sus objetivos, y la llamada justicia restaurativa juvenil (JRJ) ha 
protagonizado importantes reformas legales y procesales en varios países del mundo ${ }^{88}$. Compartiendo principios y valores presentes en diversos instrumentos internacionales que refieren a infancia y justicia juvenil, responde a la demanda por un sistema especializado centrado en la reinserción social, sumándose a la necesidad de la desjudicialización, la restricción de la privación de libertad y de enfatizar el rol de la familia, fomentando la dignidad y responsabilización del joven: Convención sobre los Derechos del Niño/a (1989); Las Reglas de Beijing (1985); Directrices de Riad (1990); Reglas de La Habana (1990); Reglas de Viena (1997).

A pesar de que la cultura en Chile se caracteriza por la judicialización de los conflictos, en los últimos años el Estado ha desempeñado un papel importante en los desafíos de trabajar con adolescentes que han cometido delitos. Esta vez, han surgido espacios fértiles para integrar nuevos paradigmas, metodologías y herramientas, como la mediación penal juvenil, una de las prácticas de justicia restaurativa. Las prácticas restaurativas se han difundido en América Latina, a través de experiencias institucionalizadas, con su propia legislación, o incluso con técnicas informales, convirtiéndose en un importante acceso a la justicia (BARACHO, 2019).

Con la entrada en vigencia de la Ley 20.084/07, Ley de Responsabilidad Penal para Adolescentes (LRPA), hubo una transición del sistema de rendición de cuentas a los jóvenes entre 14 y 17 años, ya que el Estado no cumpliría efectivamente el objetivo de rendición de cuentas establecido en el artículo 20 de la LRPA, dada la ineficiencia de las sanciones para formar parte de una amplia intervención socioeducativa, orientada hacia la plena integración social; ni respetaría ciertos principios del derecho internacional de los derechos humanos, establecidos en la Convención Internacional sobre los Derechos del Niño, y también en otros instrumentos normativos.

En ese contexto, al analizar las posibilidades de implementación de la Justicia Restaurativa en Chile, a través de la mediación penal restaurativa juvenil ${ }^{89}$, el tema se considera importante $\mathrm{y}$ actual, debido a los desafíos y perspectivas basadas en el Abolicionismo Penal de la criminología crítica de Hulsman, Nils Christie y la pensadora Elliott, con su enfoque teórico y práctico sobre la Justicia restaurativa para la estructuración de un modelo crítico para América Latina.

En otras palabras, es necesario utilizar la perspectiva abolicionista de Hulsman y Nils Christie para trabajar con la lógica del delito como situación problemática y, también,

\footnotetext{
${ }^{88}$ Ver más en: Dunkel, Horsfield y Parosanu (2015).

${ }^{89}$ Expresión utilizada en Chile.
} 
expropiar algunos conflictos de la Justicia Penal, para luego, tratar de enfrentar seriamente el encarcelamiento de jóvenes segregados y el descuido del Estado con las víctimas de un sistema reproductivo que perpetúa la lógica punitiva. Para ello, el objetivo de este artículo es observar cómo se produjo o se está produciendo la implantación de la Justicia Restaurativa en Chile; y como problema de investigación: ¿cuáles son los límites y las posibilidades de la implementación de la mediación penal juvenil en Chile en cuanto una de las prácticas de Justicia Restaurativa? Se utilizará el materialismo dialéctico como método de aproximación y técnicas de investigación documental y bibliográfica.

Este texto se divide en dos partes: la primera tratará la experiencia de la mediación penal juvenil, como una de las prácticas de justicia restaurativa; y el segundo, el sentido de justicia social en la justicia restaurativa desde la perspectiva del abolicionismo penal.

\section{La experiencia de la mediación penal juvenil en Chile}

Inicialmente, es importante mencionar la contribución histórica de la construcción y elaboración legislativa en Chile, teniendo como ley principal la Ley de Responsabilidad Penal Juvenil. La Ley N²0084/2007 (Ley de Responsabilidad Penal Juvenil), que entró en vigor el 8 de junio de 2007, por la que se establece la Mediación Penal Juvenil, propuso una importante reforma en lo que respecta a la sanción de los delitos simples, con el joven menor de dieciocho años, tratando al adolescente "[...] como un sujeto de derecho que debe ser protegido en su desarrollo e inserción social y lograr objetivos de prevención del delito" (COUSO, 2008, p. 97).

La legislación mencionada considera a los adolescentes entre 14 y 17 años como imputables. Para las autoras, la Ley de Responsabilidad Penal Juvenil también establece un sistema de garantías para los adolescentes, en el que participan actores especializados en la justicia, como: jueces, abogados, defensores públicos. También tiene sanciones penales distintas y equivalentes según la gravedad del delito (QUILODRÁN; LABRENZ; MORALES, 2018).

Para Gude (2010) la mediación penal se identifica como una práctica específica de la Justicia restaurativa, aunque la asociación entre ambos conceptos no es pacífica o unánime. En Chile, la posibilidad de desarrollar la mediación penal de manera formal o institucionalizada comenzó en el legislativo, por primera vez, con la Reforma Procesal Penal, que a partir de diciembre de 2000 entró en vigor gradualmente en las diferentes regiones del país. 
El nuevo Código Procesal Penal incorporó las llamadas Salidas Alternativas al Proceso Penal (mediación penal para adultos) que constituyen formas de término anticipado al proceso, establecidas como excepción al principio de legalidad en la persecución penal, y que implican el reconocimiento por parte del Estado de la necesidad de establecer mecanismos de solución al conflicto penal distintos al proceso penal y la pena tradicionales. Estas Salidas Alternativas consisten básicamente en la Suspensión Condicional del Procedimiento y los Acuerdos Reparatorios (GUDE, 2010, p. 06).

Durante 2015, el Ministerio de Justicia y Derechos Humanos comenzó a dirigir varios grupos de trabajo intersectoriales para discutir aspectos críticos específicos de la Ley de Responsabilidad Penal Juvenil. Discutieron la posibilidad de implementar la Justicia Restaurativa, ya que es un enfoque internacionalmente válido y reconocido, dada su armonía con la Convención y utilizada en diferentes países, incluidos los de América Latina ${ }^{90}$.

Como resultado de esta reunión, a principios de 2016, la Unidad de Coordinación y Estudios del Ministerio de Justicia y Derechos Humanos comenzó a diseñar un Estudio Práctico de mediación penal para adolescentes que cometen infracciones. La iniciativa estuvo motivada "por la necesidad de mejorar la aplicación del actual sistema de responsabilidad penal de los adolescentes (RPA) en relación con los principios de la Convención sobre los Derechos del Niño y otros instrumentos internacionales sobre justicia juvenil" (UCE, 2016). Después de varios meses de preparación, el estudio práctico comenzó a implementarse mediante la contratación de un mediador, que informaba directamente al Ministerio de Justicia y Derechos Humanos desde octubre de 2016. Las remisiones comenzaron a promoverse a partir de diciembre, llegando al primer caso en febrero de 2017 (BOLIVAR, 2017). Se corrobora sobre el proceso de implementación de la mediación penal restaurativa juvenil a través de las iniciativas de los actores sociales como fue el acuerdo de colaboración:

El compromiso fue materializado con un "Convenio" de colaboración para la derivación de casos a la mediación penal juvenil y Estudio Práctico firmado por el Ministerio Público, la Defensoría Penal Pública y el MJYDH, pensando en poder analizar la posibilidad específica de incluir procesos de mediación penal para jóvenes infractores y definir criterios y metodologías para la incorporación de un futuro Programa Nacional de Mediación Penal

\footnotetext{
${ }^{90}$ Este informe trató de discutir el incipiente proyecto sobre la Justicia Restaurativa en Chile, más precisamente la mediación penal resultante de los debates entre los miembros del Ministerio de Justicia y Derechos Humanos, que tuvo lugar a partir de 2015 y en vista de las necesidades de cambios de la legislación juvenil, para hacer frente a la reinserción social de los adolescentes. Debido a esto, comenzó la implementación de la mediación penal, en algunos casos, en el año 2016-2017. Esto fue considerado el proyecto Piloto de Chile. También como socio en el desarrollo y seguimiento de este proyecto piloto está la Universidad de Ciencias Humanas de Chile con algunos especialistas al frente, para mencionar a la investigadora y coordinadora del proyecto Daniela Bolívar y otros investigadores fuera de Chile, como el Asesor Internacional, Doctor Ivo Aertsen.
} 
Juvenil dentro del contexto de las reformas a la LRPA, específicamente en el nuevo Servicio Nacional de Reinserción Social Juvenil. Dicho documento asume el sentido estratégico de ocupar el espacio de la carencia legislativa sobre la materia en el país para efectos de validar el trabajo institucional y viabilizar la iniciativa, vinculando simbólicamente a las máximas autoridades de las instituciones, siendo la primera vez que se hace un Convenio con estas características en Chile. Dentro de este documento, se unieron ciertos criterios para su ejecución de la experiencia, fruto de la mesa de trabajo compuesta por los actores del nivel nacional de cada institución, y que constituye el "Protocolo de Derivación" de los casos. Desde la Unidad de Coordinación y Estudios perteneciente al MJYDH se comenzó a diseñar y luego de varios meses de preparación, el EP comenzó a implementarse. Desde el nivel nacional de ambas instituciones, en la misma instancia de la mesa de trabajo que resultó en el Convenio, se definió que en su primer año sería implementación en la Fiscalía Regional Centro Norte. Luego, la Defensoría Penal Pública Norte asume como la "contraparte" por ser ahí donde se tratan los casos específicamente relacionados a responsabilidad penal adolescente, que es el contexto de implementación de la experiencia. Actualmente, dicha experiencia fue expandida y también está siendo implementada en la ciudad de Valparaíso. En el EP se definió por primera vez una cobertura que promoviera el acceso a la mediación penal, de casos de mayor relevancia en comparación a las experiencias anteriores. Además, se definió un modelo de gestión y se establecieron requisitos específicos de derivación (MIRANDA, 2018, p. 57)

En abril de 2017, se promulgó la ley que crea el Nuevo Servicio de Reinserción Social e introducen enmiendas a la Ley $\mathrm{N}^{\circ}$ 20084/2017, estableciendo un servicio nacional guiado por los principios de especialidad, intersectorialidad y descentralización.

El nuevo Servicio Nacional de Reinserción Social introduce modificaciones específicamente en el ámbito del sistema de justicia criminal juvenil, esencialmente a la LRPA, adoptando dentro de sus fundamentos la creación de un programa de mediación penal como medida de diversificación reconocida. Es en este contexto de revisión de la respuesta estatal a los jóvenes infractores de ley que el Ministerio de Justicia y Derechos Humanos de Chile (en adelante MJYDH) asume la responsabilidad de promover enfoques consistentes con la $\mathrm{CDN}$ en el abordaje de la problemática, posicionando la JR y la mediación penal como una prioridad (MIRANDA, 2018, p. 57).

Según la Tabla 01, que figura a continuación, sobre los Elementos del Protocolo de Derivación de los casos de Estudio Práctico, se observa una selección de la población beneficiaria, así como el carácter leve de los delitos o faltas cometidas por los adolescentes, que pueden ser recibidos por la mediación penal restaurativa juvenil. Analizando estos recortes categóricos, dejando los delitos graves fuera de la propuesta, se percibe la fragilidad y la limitación de la práctica restaurativa. 
Tabla 1: Elementos del protocolo de derivación de casos del estudio práctico

\begin{tabular}{|c|c|}
\hline CATEGORÍAS & CONTENIDO \\
\hline & Población Beneficiaria \\
\hline Cobertura & $\begin{array}{l}\text { Jóvenes a quienes se les impute la comisión de una falta o simple delito de naturaleza } \\
\text { penal, cuya responsabilidad se rija por la Ley } 20084\end{array}$ \\
\hline & $\begin{array}{l}\text { Víctimas de faltas o simples delitos de naturaleza penal, imputados a jóvenes cuya } \\
\text { responsabilidad se rija por la Ley } 20084\end{array}$ \\
\hline & $\begin{array}{l}\text { Al menos una de las partes del conflicto deberá tener domicilio o residencia en alguna de } \\
\text { las comunas de competencia de la Unidad de Justicia Vecinal de Norte (Renca, } \\
\text { Independencia, Cerro Navia, Pudahuel y Quilicura) o Poniente (Estación Central) }\end{array}$ \\
\hline & Espacial \\
\hline & $\begin{array}{l}\text { Territorio correspondiente a la Fiscalía Regional Metropolitana Centro Norte (en } \\
\text { adelante FRMCN) }\end{array}$ \\
\hline Natural & de la infracción: falta o simple delito. Se excluyen expresamente los siguientes: \\
\hline Requisitos de & Delitos dolosos contra la vida \\
\hline derivación & Delitos contra la libertad ambulatoria \\
\hline & Delitos contra la libertad sexual cometidos contra personas menores de edad \\
\hline & $\begin{array}{l}\text { Delitos y faltas tipificados en la ley } 20000 \text {, a excepción de los previstos en los artículos } 4 \text {, } \\
8 \text { y } 50 .\end{array}$ \\
\hline & Delitos cometidos en contexto de violencia intrafamiliar. \\
\hline & $\begin{array}{l}\text { Infracciones penales que involucren el uso de armas y/o violencia en las personas, } \\
\text { aquellos en que el infractor tenga condenas previas y cuando el infractor no se encuentre } \\
\text { escolarizado o no esté dispuesto a hacerlo como condición previa para la derivación o } \\
\text { como parte de una condición de una suspensión condicional del procedimiento posterior a } \\
\text { la mediación }\end{array}$ \\
\hline
\end{tabular}

Fuente: Miranda, 2018.

En Chile existe una institución encargada de implementar los programas de reparación de daños, y tiene cuatro de ellos distribuidos en diferentes regiones del país. Cada uno de ellos tiene un dúo psicosocial formado por un trabajador social (Asistente Social) y un psicólogo responsable del proceso. En una entrevista realizada por las autoras, se encontró que en Chile no existe un programa específico y especializado en mediación penal juvenil; y en el programa de reparación de daños, los entrevistados mencionaron que reciben en promedio cinco casos por año, así como informaron que los fiscales, abogados, defensores y jueces aplican frecuentemente la sanción penal al adolescente y que no es su práctica remitir los casos a la institución responsable. Una de las dificultades que enfrenta el programa es la distribución geográfica de los casos, en un territorio de 16000 kilómetros cuadrados (QUILODRÁN; LABRENZ; MORALES, 2018).

Además, los mismos profesionales atienden los casos en el programa de servicios en beneficio de la comunidad, también dirigidos a los adolescentes. Sin embargo, y porque son un grand número de casos, alrededor de 20, perjudica la calidad de la atención, especialmente en el programa de reparación de daños, donde involucra la práctica víctima 
delincuente en la mediación. El retraso en el contacto con el adolescente y la continuidad con el trabajo debilita el resultado de la restauración. El perfil de los adolescentes es amplio, algunos tienen una gran implicación con el crimen, otros poca, las familias son vulnerables y no participan en el programa. Por lo tanto, el sistema chileno es punitivo, hace visible la víctima y el adolescente.

En resumen, en Chile no existen centros que aborden específicamente la mediación entre los adolescentes y las víctimas. Las prácticas restaurativas están aisladas. Unas de las razones es la naturaleza punitiva del sistema penal de Chile. Para la recepción de la Justicia restaurativa, el sistema penal de Chile necesita escuchar el adolescente e invertir en políticas públicas de prevención, abriéndose a las posibilidades contemporáneas y humanistas (QUILODRÁN; LABRENZ; MORALES, 2018). En el mismo sentido:

El desarrollo de este tipo de práctica aún son tímidas en el país, persistiendo el desafío de trabajar en una política nacional tendiente a hacerla efectiva, como lo que ha sucedido en el caso de la mediación familiar (Díaz, 2010). La mediación como la práctica restaurativa más implementada en el contexto chileno, originalmente estuvo más bien conectada a una técnica de resolución de conflictos en la esfera civil y comunitaria, trasladándose luego hacia el ámbito penal (Díaz \& Navarro, 2015). Muchas de esas iniciativas de mediación han sido entendidas como "restaurativas" (Díaz, 2010; Díaz \& Navarro, 2015), lo que establece un importante avance de las concepciones sobre mediación penal en el medio nacional, que, a pesar de su origen en el contexto de familia, civil y comunitario, camina hacia las concepciones propias de la justicia restaurativa (Díaz, 2010; Díaz \& Navarro, 2015).[...] Además, se indica que los programas implementados en Chile hasta ahora, por lo general no consideran la participación del entorno social, la familia y la sociedad civil (González \& Fuentealba, 2013) (MIRANDA, 2018, p. 35).

Debido a estas circunstancias en particular, de la necesidad de desarrollar la mediación penal restaurativa juvenil, refutando el carácter punitivo con el adolescente, el proyecto desarrollado por el Ministerio de Justicia y Derechos Humanos sugirió en su primer análisis algunas recomendaciones, entre ellas: la continuidad, divulgación y multiplicación de los programas y prácticas de mediación y otros modelos como las conferencias, ampliando, seleccionando, apoyando y registrando el número de casos que se abordarán en el programa de mediación existente, con el propósito de estandarizar y calificar la atención en un corto período de tiempo (BOLIVAR, 2017).

Debido a esto, el Ministerio de Justicia se preocupó por elaborar un sistema de supervisión para la mediación penal que se recibirá en el Nuevo Servicio de Reinserción Social Juvenil. Los objetivos específicos son: elaborar una propuesta de normas técnicas para 
el servicio prestado; diseñar un instrumento de evaluación de los procesos y la calidad de la mediación penal y elaborar directrices para implementar los procesos de supervisión de las prácticas de justicia restaurativa (MIRANDA, 2018).

Desde el análisis conceptual a través de la literatura nacional e internacional, destacando las normas internacionales y las latinoamericanas con una perspectiva restaurativa en el contexto juvenil, se proyectaron los ocho (8) pasos para la implementación de la práctica restaurativa para los jóvenes en Chile. Cabe señalar que el país chileno se encuentra entre los pasos 7 y 8 , es decir, en el paso 7: sensibilizar y convencer a los dirigentes políticos para la continuidad del proyecto. $\mathrm{Y}$ en el paso 8: la sostenibilidad. Por lo tanto, es necesario establecer indicadores de calidad y supervisión para mantener la práctica y convertirse en una política pública (MIRANDA, 2018).

Basado en el Observatorio Internacional Justicia Juvenil, se adoptó el modelo holístico que consta de 4 niveles; reconociendo en el tercer nivel la implementación de la mediación penal juvenil como una práctica o política de prevención para los jóvenes, con el objetivo de evitar su contacto con el sistema penal criminal. Estos modelos fueron adoptados para rediseñar una política pública que no sea punitiva, sino que responda al adolescente chileno.

Sin embargo, la cuestión es si es posible proponer la mediación penal restaurativa juvenil dentro de la Ley de Responsabilidad Penal. Al rediseñar y pensar en el nuevo Servicio de Reinserción Social Juvenil, será necesario abordar los pasos, niveles y desafíos, dando seguimiento a los puntos elaborados y sugeridos en el informe final a solicitud del Ministerio de Justicia y Derechos Humanos de Chile.

En concreto, el análisis acerca de la implementación de la ley no ha sido positivo, apuntando al no cumplimiento de su objetivo y la inefectividad de ciertas sanciones, centrándose más en la individualización de la culpa y se distanciando del carácter "restaurativo", con graves falencias en el diseño y aplicación de algunas sanciones (Boletín N S 1.555-14, 2012; Comité de Evaluación OCDE, 2015): la sentencia condenatoria es la salida más habitual para los jóvenes y, a pesar de la disminución del uso de la privación de libertad, hubo un aumento importante en la duración de las condenas de privación de libertad (BERRÍOS, 2011). Evaluada en distintos momentos, distintas comisiones e instituciones relatan que la implementación de la Ley no ha sido prioridad del Estado, con escasa coordinación entre el sistema de intervención y el sistema legal, destacándose (MIRANDA et al, 2018): la falta de especialización y capacitación de los profesionales en materia infantojuvenil; y la falta de condiciones y ofertas programáticas en los recintos que están a 
cargo de implementar las sanciones, a través del llamado Servicio Nacional de Menores (SENAME), sobre la gestión del Estado .

Sobre la Política de Infancia que adopta el Estado de Chile, es muy controversial en el trato y cuidado con sus niños, niñas y adolescentes, ya que carece de una legislación de protección integral a la infancia, pero, por otro lado, concretizó una LRPA; mejor diciendo, interviene solamente cuando se presenta una situación de vulneración o de irregularidad, desde donde el Fondo de las Naciones Unidas para la Infancia, en la Agenda de Infancia 2018-2021, establece el principal desafío de Chile: la creación de un Sistema de Protección Integral de Derechos de la Niñez (MIRANDA et al, 2018).

Por otro lado, frente a las deficiencias del Estado, y en el sentido de ratificar su compromiso con la CDN se presentan diversos proyectos ley: en 2015 se presentó la propuesta que trata del Sistema de Garantías de los Derechos de la Niñez, en el sentido de proteger y garantir de manera integral el ejercicio efectivo y el goce pleno de los derechos de los niños y, específicamente en el contexto penal juvenil, en 2017 se presentó el proyecto de ley que crea el nuevo Servicio Nacional de Reinserción Social Juvenil (SNRSJ), ambos en tramitación actualmente.

El Sistema de Garantías de los Derechos de la Niñez está pautado en principios como de la autonomía progresiva y del interés superior del niño, y establece como orientadores de las políticas de Estado la participación y el derecho a ser oído, abriendo espacio para reformar aspectos problemáticos en el sistema penal juvenil (BOLÍVAR et al., 2017). En ese contexto, y esencialmente a la LRPA, el nuevo SNRSJ propone una reforma no solo desde un punto de vista procesal, sino que también estructural, diseñando una política consistente de reinserción social y un servicio descentralizado, que asume un carácter pionero en el país. Contempla un modelo de intervención basado en los principios de la Justicia Restaurativa (JR), entre otras perspectivas teóricas, y propone la creación de un Programa de Mediación Penal como medida de diversificación y nueva vía de alternatividad al proceso, proyectando una mejora en la calidad de la respuesta estatal al conflicto (Boletín 11174-07).

En concreto, asumiendo la JR como un enfoque teórico y una metodología que ha sido validada por la evidencia, Chile está comenzando a integrarse en el desafío de incorporar la JR en la compresión del conflicto penal y los procesos de reinserción social de sus jóvenes, pero respondiendo también a los derechos y necesidades de las víctimas. Dicha iniciativa nacional responde a la recomendación de organismos internacionales y regionales acerca de la inclusión de la JR en los procedimientos penales, especialmente en la justicia juvenil , y además está en consonancia con la Política Nacional de Víctimas que, respondiendo a 
normativas regionales e internacionales, establece la "necesidad de avanzar en la generación de nuevas opciones de justicia, como por ejemplo, la justicia restaurativa" (Subsecretaría de Prevención del Delito, 2016, p. 13), enfocándose en la reparación del daño, las necesidades y los derechos de las víctimas de delitos.

En general, las prácticas restaurativas en el país son escasas, de las cuales la mediación se destaca y si bien estuvo originalmente relacionada a la esfera civil y comunitaria, viene ganando espacio en la penal, caminando hacia las concepciones propias de la JR (DÍAZ, 2010; DÍAZ; NAVARRO, 2015). Sobre la implementación de la mediación penal propiamente, desde el año 2000 fueron al menos 20 iniciativas desarrolladas en Chile, las cuales en su mayoría se dieron en el contexto de justicia de adultos, con poca sistematización y evaluación, y utilizaban protocolos de otras realidades legislativas o adecuando metodologías utilizadas en la mediación familiar (regulada legalmente en Chile) (DÍAZ; NAVARRO, 2015; GONZÁLEZ; FUENTEALBA; 2013).

La primera experiencia sistematizada de mediación penal juvenil en el país es el denominado Estudio Práctico (EP en adelante). El Ministerio de la Justicia y Derechos Humanos (MJYDH), frente a la necesidad de (1) analizar la posibilidad específica de incluir procesos de mediación penal para jóvenes infractores y (2) definir criterios y metodologías para la incorporación de un futuro Programa Nacional de Mediación Penal Juvenil dentro del contexto de las reformas a la LRPA en el nuevo SNRSJ, decidió ejecutar una experiencia piloto de mediación penal en el contexto penal juvenil. El EP fue puesto en práctica en enero de 2017 y hasta la presente fecha de realización está aún en funcionamiento.

Para materializar la derivación de casos a la mediación penal juvenil y EP, el Ministerio Público, la Defensoría Penal Pública y el MJYDH firmaron un Convenio de Colaboración que, además de unir criterios para la ejecución de la experiencia, viabiliza la iniciativa por lograr vincular simbólicamente a las máximas autoridades de las instituciones, siendo la primera vez que se hace un Convenio con estas características en Chile. Ese documento tiene especial relevancia ya que el país aún carece de ley expresa para respaldar las prácticas de la JR, fomentando la internalización del tema entre los operados, de sus principios, estándares y buenas prácticas.

En concreto, el EP, además de cuidar en resguardar los principios de la JR, responde a enseñanzas dejadas por experiencias anteriores implementadas en el país (DÍAZ; NAVARRO, 2015): Establece una buena coordinación entre los actores involucrados, garantizando la activa participación de las instituciones (Ministerio Publico y Defensoría Penal Pública, sobre la gestión del MJYDH), la confianza y el compromiso entre los actores 
involucrados de todos los niveles; Promueve un estudio que ofrece asesoría, capacitación a los actores, y además evalúa su primer año de implementación, con espacio para corregir debilidades durante ese proceso

Diseña e implementa buenos protocolos de actuación entre los centros de mediación y el Ministerio Publico, asegurando la derivación de casos y relación directa y rápida entre las instituciones; Criterios de derivación definidos y que consideran, por primera vez, la accesibilidad de casos con mayor relevancia jurídica (experiencias anteriores de derivación de casos fue incipiente y esporádica, y en su mayoría delitos de bagatela, principalmente en delitos de orden patrimonial); Considera la participación del entorno social, la familia y la sociedad civil, y principalmente considera la relevancia de la víctima y su red de apoyo en el proceso.

Si bien durante su primer año su implementación se produjo específicamente en la ciudad de Santiago, región metropolitana de Chile, objetivamente casos tratados en la Fiscalía Regional Centro Norte, actualmente fue expandida y también está siendo implementada en otras ciudades del país (Valparaíso y La Serena). Esa expansión ocurrió como respuesta a los resultados positivos del proyecto, el cual también sirvió como antecedente importante para la propuesta de un futuro Programa de Mediación Penal Juvenil en el marco del nuevo Servicio Nacional de Reinserción Social, actualmente en tramitación en el congreso.

\section{El sentido de la justicia social y su interfaz con el abolicionismo penal}

El enfoque conceptual de la Justicia restaurativa en Chile y en algunos países especialmente en América Latina, cambia de un lugar a otro, pero no deja dudas sobre la lógica de romper con la retribución de la pena y buscar restablecer el diálogo, el respeto al otro en las relaciones humanas. Sin embargo, hay algo que todavía no está claro y no se ha superado, que es la necesidad o el sentido de justicia social en este contexto, que también necesita ser enfrentado por los adeptos a las prácticas restaurativas. Elliott menciona a Ruth Morris en su trabajo sobre el tema, criticando más específicamente "la teoría de la Justicia restaurativa por su falta de atención a las cuestiones de justicia social" (ELLIOTT, 2018, p. 249)

En este contexto, existe también la correlación con el abolicionismo penal, ya que defiende la propia extinción del sistema punitivo, incluso en su forma más radical, que refuta toda la forma de castigo, porque ¿cómo responsabilizar a los adolescentes en un país hecho de injusticias sociales? (ELBERT, 2009). Las opiniones simplistas del derecho penal no contemplan la complejidad de la vida humana, donde, según Achutti y Leal (2017), el 
enfoque en los actos y no en las interacciones crea una visión distorsionada y estancada que no coincide con la realidad, porque las relaciones son dinámicas, incluso en relación con la posición de víctima y delincuente. Por lo tanto, se entiende que el castigo tiene que ver con la imposición intencional de daños por agentes autorizados para ello - ya sea el Estado, el director de la escuela o los padres. El castigo denota la imposición de un dolor injustificado en el contexto de una sociedad regida por el Estado de Derecho. En cuanto al deseo colectivo de castigar, esto, por supuesto, precede a la capacidad penal de la ley para castigar. En otras palabras, la justicia no puede reducirse a la ley, al derecho o al sistema retributivo, ya que no es un problema jurídico sino un problema humano (ELLIOTT, 2018). Especialmente de un tema multidimensional: físico, político, económico, espiritual y social. Por lo tanto, el yo y sus relaciones (SALM, 2017).

Revelar la realidad puesta en el ápice abisal, que implica la desigualdad social, es trabajar más allá de sus consecuencias en las instituciones judiciales y las penitenciarías, por ejemplo, es desvestirse de la naturalización presentada sobre la realidad social. La realidad social naturalizada hace que las personas o sujetos sean incapaces de percibir la dimensión compleja e intersubjetiva en las prácticas de control, dominación y distinción de clases (SOUZA, 2018).

Asimismo, pensar desde esta perspectiva es recordar y comprender las implicaciones y consecuencias de la desigualdad social en el sistema penitenciario, así como las categorías construidas por el Derecho Penal contemporáneo que atiende al sistema de justicia actual, sirviendo como herramienta deshumanizadora y de reproducción de las más diversas violencias contra el ser humano. Por lo tanto, al buscar la Justicia restaurativa como otra forma de pensar y vivir la justicia en las relaciones, se desea un derecho integrador y no con una lógica punitiva, reproduciendo meramente la discriminación racial y los campos de aislamiento y segregación de las minorías sociales. (ACHUTTI, 2014).

La Justicia restaurativa no puede ejercerse simplemente en la sistematización de la Justicia Criminal, ni en ninguna otra sistematización. Debe representar quiénes somos, así como la respuesta que damos a los problemas cotidianos, buscando una mayor independencia de las instituciones gubernamentales para tratar ciertos conflictos, haciendo así el ciudadano más comprometido con la comunidad y competente para tratar de manera justa en una posible confrontación. (ELLIOTT, 2018).

Los abolicionistas Hulsman y Nils Christie consideran que el sistema penal interviene en muchos puntos que las personas involucradas no experimentan como delitos, olvidándose de la víctima en el proceso. También sostienen que la intervención del sistema 
penal agrava los conflictos y opera según la lógica del castigo, porque impide cualquier respuesta que no sea punitiva. Reconocen que el encarcelamiento es un caldo de cultivo para el crimen, y es sólo una alternativa a la tortura y la pena de muerte. Significa: "En la cárcel, los hombres son despersonalizados y dessocializados" (HULSMAN; CELIS, 1993, p. 63).

En cualquier caso, el discurso abolicionista es de reformar la culpa y el castigo, ya que se sabe que no se regenera, ni atribuye efectivamente la responsabilidad individual y colectiva. En este contexto, se reconoce que el abolicionismo no es un nuevo paradigma sino una teoría, ya que sirve para sensibilizar y generar mayores preguntas sobre lo que se pone en el campo social, retroalimentado por la cultura jurídica punitiva y no declarada (CHEERER; CHRISTIE, 1989). De hecho, Hulsman (1993) no le ve sentido en las sanciones, sobre todo porque cree que la administración de los conflictos por parte del Estado necesita ser repensada, abolida, por cierto, descentralizada, es decir, quitarle al poder público la autonomía en la regulación de los conflictos, porque la estructura de las sanciones sólo retroalimenta el poder simbólico del sistema penal. Se necesita un sistema de gestión de conflictos más autónomo, similar al de algunas comunidades tribales, que regule los conflictos a nivel de intergrupos y las relaciones directas entre individuos con la ayuda de instituciones o procedimientos cercanos, y que observen la experiencia directa de las personas a fin de trabajar con la dimensión de la ética, el cuidado de los demás y la responsabilidad de los actos decididos y adoptados por el individuo.

Es fundamental retomar el pensamiento abolicionista para proponer otras modalidades de administración de conflictos en la justicia criminal. Por lo tanto, la Justicia restaurativa puede ser otra posibilidad para repensar y enfrentar la política de encarcelamiento. (ACHUTTI, 2014, p. 67).Para Ballesteros, los programas y las experiencias de Justicia restaurativa no se limitan al área de la justicia penal. Sin embargo, no es suficiente su implementación dentro del sistema penal, es necesario transformarlo, porque la Justicia restaurativa tiene sus propios valores y principios (BALLESTEROS. 2018).

Entonces, en el mismo momento en que surge esta posibilidad de trabajar con la Justicia restaurativa en la justicia penal, y no sólo con la justicia juvenil, se perciben los limites restaurativos, basados en conceptos sobre la Justicia restaurativa, cuando reducen la complejidad de la práctica judicial o extrajudicial a un proceso. Sin embargo, la Justicia restaurativa tiene límites. Algunos de los desafíos que se deben enfrentar son: la desigualdad social (racismo, condición económica, feminismo, patriarcado), el encarcelamiento masivo, el 
conservadurismo judicial (tradición-seguridad jurídica, poder, baja integración del poder Judicial con la sociedad civil (centros comunitarios).

Caminar en esta dirección impone necesariamente un pensamiento colectivo que cuestione a todos los actores comprometidos con el proceso de cambios y los conceptos positivistas y punitivos, especialmente las mitologías que no se adaptan al paradigma emergente. Este es un requisito que tiene profundas implicaciones prácticas en todo el edificio restaurativo, que no se consolidará plenamente si no supera, estructuralmente, los antiguos cimientos y si no cumple ciertos requisitos, que son a la vez epistemológicos y políticos. ${ }^{91}$ Como ejemplo en Brasil, los desafíos con la Justicia restaurativa, entre tantas cosas mencionadas anteriormente, necesitan superar los significados y (pre)conceptos del positivismo criminológico y jurídico, adoptando otro vocabulario. Además, debe superar la comprensión de ser sólo un método resolutivo de conducta poco ofensiva (BRASIL, 2018). Por lo tanto, ¿cómo pensar en un modelo ideal de Justicia restaurativa en Chile y América Latina?

\section{Conclusión}

En ese sentido, conocer la experiencia de Brasil y Chile (considerando las fortalezas y los desafíos), abre un espacio importante de aprendizajes para aquellos contextos en que la implementación de las practicas restaurativas aún están en una etapa temprano de implementación, como Chile, pero también ayuda a visualizar con mayor claridad elementos que deben ser revisados en países con experiencia más avanzada, como propiamente Brasil.

Desde el nivel de las políticas públicas sobre la infancia, se observa que ambos países tienen debilidades significativas en la impugnación de las recomendaciones internacionales que ratifican el enfoque de los resultados, existe una escasez de espacios de participación más amplios por parte de estos actores, que se limitan a la asistencia y carácter tutelar, como objetos necesitados y bien nada capacidades de resocialización. Por otro lado, fortalece la necesidad de difundir las prácticas restaurativas en el contexto de la infancia, y puede presentarse a los jóvenes en situación de victimización, testamento o delincuente, y promoverse en todas las etapas del proceso de justicia penal.

\footnotetext{
${ }^{91}$ En este sentido, son fundamentales los aportes de Christie (1977), Hulsman (1993; 1997) y Baratta (1987; 1991; 1993), que trabajan positivamente en una agenda de superación de la cultura y el paradigma punitivo, con modelos que incluyen desde principios para la minimización de la violencia en el sistema penal hasta principios y propuestas para la construcción alternativa de los problemas sociales, sin olvidar la primacía a las víctimas y los mecanismos restaurativos. En cuanto al enfoque del abolicionismo penal de Christie y Hulsman, ya ha trabajado Achutti (2014).
} 
Este artículo tuvo como premisa inicial presentar los resultados parciales de un estudio de pasantía de doctorado sobre las posibilidades, obstáculos, desafíos y perspectivas para implementar la Justicia restaurativa o mediación penal juvenil en Chile, desde el punto de vista del abolicionismo penal.

La experiencia de Chile, aunque incipiente, obtenida en los últimos años, tiene mucho que decir y ser explorada por otros países de América Latina, especialmente Brasil, sobre cuál es el significado y la utilidad de la Justicia restaurativa en los conflictos, así como, profundizar y enfrentar la cuestión de la Justicia social. En esta área, la pregunta era: ¿cuáles son los límites y las posibilidades de la implementación de la mediación penal juvenil en Chile en cuanto una de las prácticas de Justicia Restaurativa?

El primer capítulo trató de la experiencia de la mediación penal en Chile, teniendo la ley de Responsabilidad Penal Juvenil como la base de su aplicabilidad jurídica. Con respecto a la legislación juvenil chilena, esta debe ser revisada y evaluada, ya que la falta de especialización para jueces y fiscales, así como el paradigma punitivo en relación con los adolescentes, dificultan la conexión y el diseño institucional para la implementación y aplicación de las prácticas de Justicia restaurativa en el País. Aunque el Ministerio de Justicia y Derechos Humanos tiene un convenio con la Universidad Católica Chilena para la elaboración de la política del Nuevo Servicio de Reinserción Social, a través de la mediación penal restaurativa juvenil, los obstáculos no se agotan en esta etapa. Es necesario un trabajo continuo de sensibilización, capacitación y formación de personas, actores o protagonistas directamente vinculados con la población juvenil y beneficiaria.

En el segundo capítulo, basado en la reflexión abolicionista, se reforzó la necesidad de revisar y ampliar el sentido de justicia para lo social. Por lo tanto, corrobora la comprensión de pensar acerca de enfrentar problemas como la desigualdad social (racismo, condición económica, feminismo, patriarcado), el encarcelamiento masivo, el conservadurismo judicial, el acentuado enfoque en la Justicia Penal Juvenil, con poca o ninguna repercusión en la justicia de adultos, y la fragilidad de la educación jurídica con otras posibilidades de gestión de conflictos.

Aunque Chile busque la humanización de la justicia juvenil a través de vías alternativas de pacificación de conflictos, no es posible un camino consolidado sin transformaciones profundas en la sociedad y en el Estado, a través de políticas públicas efectivas y no compensatorias.

\section{Referencias}


Achutti, D.; Leal, M. A. S. Justiça Restaurativa no Centro Judiciário de Solução de Conflitos e Cidadania: da teoria à prática. Revista de Criminologia e Políticas Criminais, Maranhão, v. 3, n. 2, p. 84-100, jul./dic. 2017.

Achutti, Daniel Silva. Is a Critical Model of Restorative Justice Possible? A Penal Abolitionist Approach. In: Aertsen, I.; Pali B.; de (Org.). Critical Restorative Justice. Blooms Bury, 2017.

Achutti, Daniel Silva. Justiça restaurativa e abolicionismo penal. São Paulo: Saraiva, 2014.

Azevedo, R. G. O movimento da informatização da justiça. In: Carvalho, S; Wunderlich, A. (Orgs). Diálogos sobre a justiça Dialogal Teses e antíteses sobre os processos: De informalização e privatização da Justiça penal. Rio de Janeiro: Lumen Juris, 2002. p. 65-69.

Ballesteros, Alejandra Mera González. Justicia restaurativa y proceso penal garantías procesales: límites y posibilidades. Revista Ius et Praxis, Chile, a. 15, n. 2, p. 165-195, 2009.

Baracho, Bianca. Profissionais jurídicos e Acessibilidade na Justiça Restaurativa: alternativa real ou mecanismo de controle? Reflexões desde a experiência de mediação penal no Chile. Revista Brasileira de Políticas Públicas, Brasília, v. 9, n. 3 p. 189-210, 2019.

Bolívar, D. et al. Estudio Proyecto Capacitación, Asesoría y Estudio Práctico Mediación Penal Juvenil. Informe Final. Santiago: Facultad de Ciencias Sociales, Universidad de Chile, 2017.

Bolívar, Daniela (Coord). Capacitación, asesoría y estudio práctico mediación penal juvenil. Informe final. Chile: Facultad de Ciencias Sociales - Universidad de Chile, oct. 2017.

\section{Brasil, CNJ. Relatório Analítico Propositivo Justiça Pesquisa Direitos e Garantias}

Fundamentais: pilotando a justiça restaurativa: o papel do Poder Judiciário. Disponible en: http://www.cnj.jus.br/files/conteudo/arquivo/2018/06/48a1d20e9350d40373889719054070b0.pdf. Acceso en: 01 julio 2019.

Brasil. Presidência da República. Secretaria Geral e Secretaria Nacional da Juventude. Mapa do encarceramento: os jovens do Brasil. Brasília: Presidência da República, 2015.

Carvalho, Camilo de Oliveira. Mediação penal não paralela e integrada à justiça restaurativa: uma proposta de efetivação do acesso à justiça no Brasil, 2014. Disertación (máster) - Universidade Federal da Bahia, Faculdade de Direito, 2014.

Christie, Nils. Conflicts as Property. British Journal of Criminology, Delinquency and Deviant Social Behaviour, Inglaterra, v. 17, n. 1, enero 1977.

Couso, Jaime. Justicia y derechos del niño. Notas para un estudio sobre la especialidad del derecho penal y procesal penal de adolescentes: el caso de la ley chilena. Colombia: Unicef, 2008 .

CPP. Centro de Políticas Públicas. Sistema carcelario en Chile: propuestas para avanzar hacia una mayor efectividad y reinserción. Pontificia Universidad Católica de Chile, 2017. Disponible en: politicaspublicas.uc.cl/wp-content/uploads/2017/05/Artículo-Sistemacarcelario-en-Chile.pdf. Acceso en 01 julio 2019. 
Elliott, Elizabeth. Segurança e cuidado: justiça restaurativa e sociedades saudáveis. São Paulo: Palas Athena, 2018.

Flores, A. P.; Roliano, M. G. O programa Justiça Restaurativa do Estado do Rio Grande do Sul: um ponto de partida ou de chegada? Disponível em: https://pitangui.uepg.br/eventos/justicarestaurativa/ANAIS2016.php. Acesso em: 08 nov. 2019.

Gomes, Daniel. IDH mostra o Brasil distante do 'pódio' do desenvolvimento humano. Jornal O São Paulo, 2017. Disponible en:

https://www.fespsp.org.br/upload/usersfiles/Paulo\%20Silvino.pdf. Acceso en: 01 julio 2019.

Hulsaman, L.; Celis, J. Penas perdidas: o sistema penal em questão. Niterói: Luam, 1993.

Human Development Reports. Human Development Indices and Indicators. Statistical Update. 2018. Disponible en:

http://hdr.undp.org/sites/default/files/2018_human_development_statistical_update.pdf>. Acxeso en: 01 julio 2019.

Mera, Alejandra. Reforma a la justicia penal adolescente: ¿Por qué no dar un giro hacia la justicia restaurativa? Santiago de Chile: Anuar Quesille, 2018.

Miranda, Paula (Coord.). Elaboración de un sistema de supervisión para la mediación penal en el marco del nuevo servicio de reinserción social juvenil. Informe final. Chile: Facultad de Ciencias Sociales - Universidad de Chile, oct. 2018.

Quilodrán; C. R.; Labrenz, C.; Morales, G. D. Justicia Restaurativa en Sistemas de Justicia Penal Juvenil Comparado: Suecia, Inglaterra, Italia y Chile. Revista Política Criminal, Chile, v. 13, n. 25, p. 626-649, jul. 2018.

Scheerer, H.; Christie, S. Abolicionismo penal. Buenos Aires: Ediar, 1989.

Data de recebimento: 18.03 .2020

Data de aprovação: 20.07.2020 